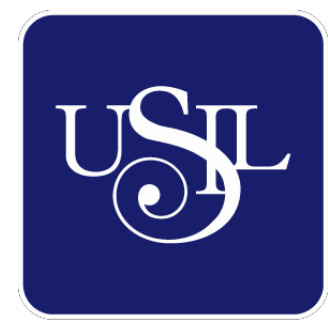

UNIVERSIDAD

SAN IGNACIO

DE LOYOLA

ESCUELA DE POSTGRADO

Maestría en Ciencias Empresariales

\title{
PLAN ESTRATÉGICO SAN MATEO
}

Trabajo de Investigación para optar el Grado de Maestro en Ciencias Empresariales

\section{JAIME LUIS ALDAVE BAZALAR \\ JESSICA MILAGROS MONTERO QUISPE \\ LUIS JAVIER NEYRA GORRITI \\ DELMY DURMAN TICSE CHOQUE}

Asesor:

Edmundo Rafael Casavilca Maldonado

Lima - Perú

2018 


\section{RESUMEN EJECUTIVO}

El presente plan estratégico, consiste en el planteamiento de las mejores estrategias que ayudarán a agua mineral San Mateo a incrementar su volumen de negocio por encima del mercado y generar mayor valor para la empresa Unión de Cervecerías Peruanas Backus y Johnston S.A. (Backus) en un horizonte de 3 años.

En la actualidad, a nivel mundial existe una tendencia creciente hacia una alimentación saludable. Esto debido a una mayor conciencia de las personas sobre el cuidado de la salud, lo que implica un cambio positivo en los hábitos de consumo y estilos de vida. Una consecuencia de este cambio ha sido que las personas comiencen a migrar del consumo de bebidas azucaradas hacia las aguas embotelladas.

El Perú no es ajeno a este cambio y los indicadores de consumo del mercado nacional lo reflejan. Tal es el caso del mercado nacional de bebidas, donde se viene experimentando un crecimiento importante y sostenido en los últimos años.

La realización del presente trabajo toma como punto de partida la información existente sobre el mercado peruano de bebidas, así como el estudio de los factores sociales, culturales y políticogubernamental. La investigación efectuada ha involucrado el análisis de fuentes secundarias tales como: datos procedentes del Ministerio de la Producción, información de CCR, estudios al consumidor de Millward Brown, Arellano Marketing y la misma empresa Backus. Con esta data se procedió a obtener información de fuentes primarias y para ello se emplearon técnicas cualitativas tales como entrevistas en profundidad a los expertos del sector, entre ellos al Gerente de marca de agua mineral San Mateo, la misma que actualmente no es percibida como agua mineral de manantial, bajo cumplimiento de precio en el punto de venta y baja penetración en la región sur y oriente. 
En el horizonte planteado de 3 años, el plan estratégico proyecta alcanzar un incremento del 40\% sobre el margen de contribución actual que Agua Mineral San Mateo aporta a la empresa Backus. Esto se logra con el conjunto de estrategias propuestas en el presente trabajo, las cuales están enfocadas en construir el beneficio diferencial de Agua Mineral San Mateo, ampliar su desarrollo regional en el País, y asegurar una correcta arquitectura de precios contra la competencia. Esto demanda una inversión en marketing de S/. 3,925,326.

Finalmente, el análisis financiero proyectado arroja un costo de oportunidad del $9.29 \%$, un WACC de $12.32 \%$, un VAN positivo y un TIR de $146.43 \%$. Estos resultados permiten concluir que la aplicación de las estrategias es viable y rentable para Agua Mineral San Mateo, por lo que se recomienda la implementación del presente plan estratégico. 


\section{Tabla de Contenido}

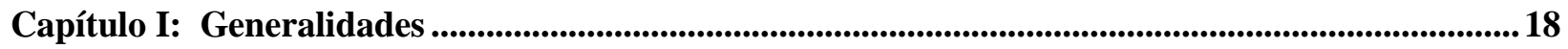

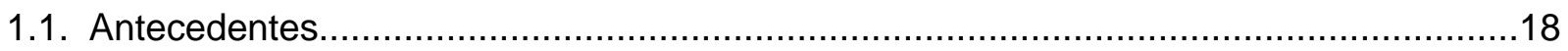

1.2. Determinación del Problema u Oportunidad .......................................................19

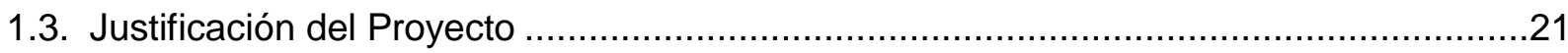

1.4. Objetivos Generales y Específicos del plan estratégico ........................................27

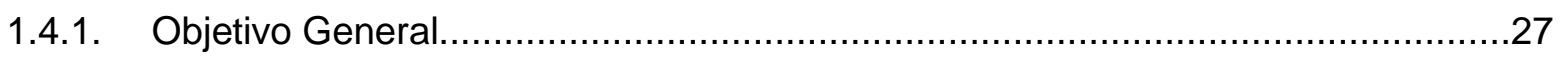

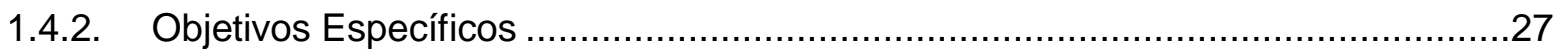

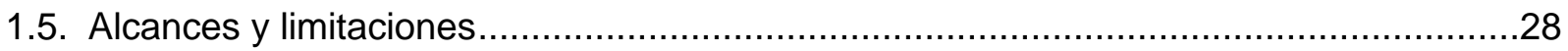

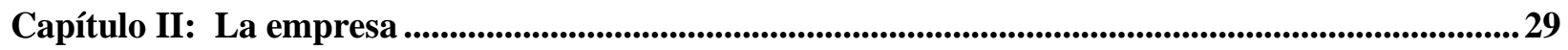

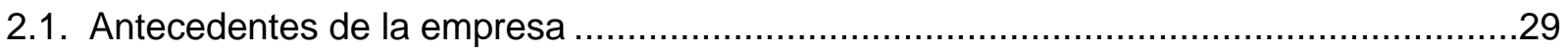

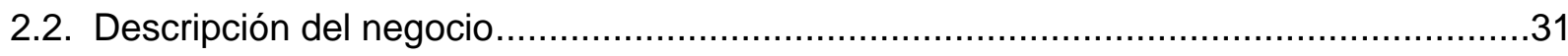

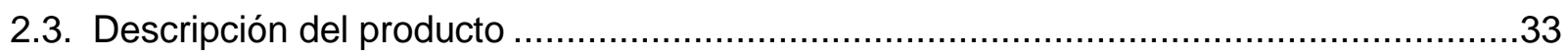

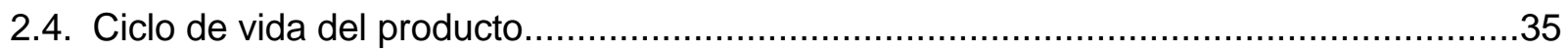

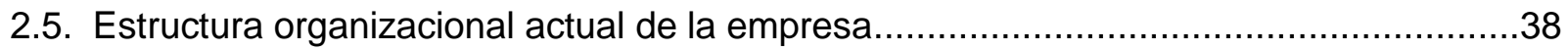

2.6. Situación de Mercado y Financiera actual de la Industria ......................................43

Capítulo III: Formulación de la Misión, Visión y Valores de la Empresa.............................................49

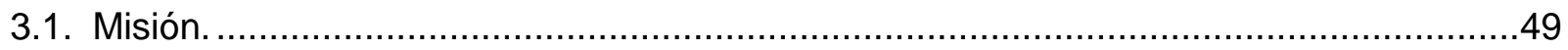

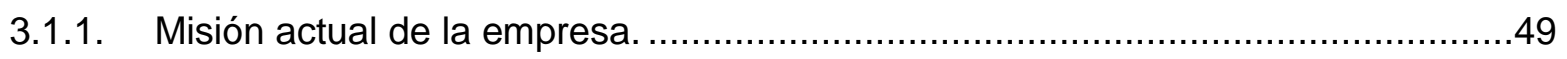

3.1.2. Análisis de la misión actual..............................................................49

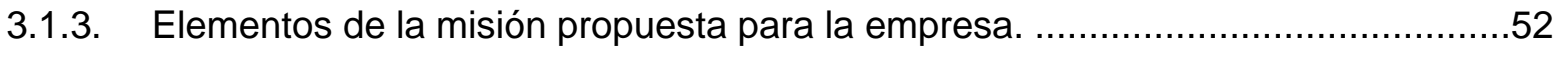

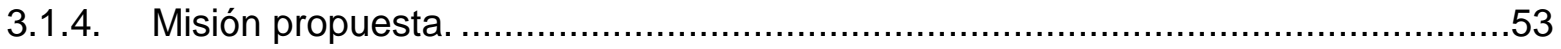

3.1.5. Matriz de la misión propuesta para agua mineral San Mateo.............................54

3.1.6. Misión propuesta para agua mineral San Mateo...............................................55 


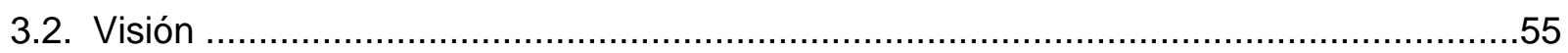

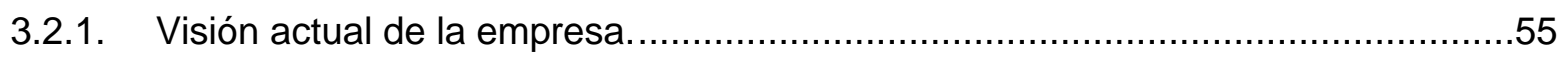

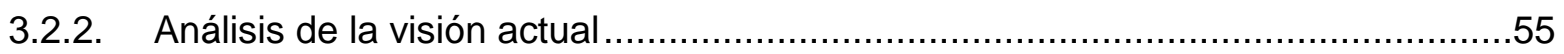

3.2.3. Matriz de la visión propuesta para la empresa Backus ..................................57

3.2.4. Visión propuesta para la empresa. ............................................................57

3.2.5. Matriz de la visión propuesta para agua mineral San Mateo.............................58

3.2.6. Visión propuesta para agua mineral San Mateo. .............................................58

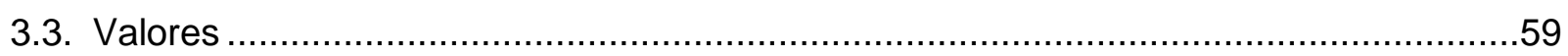

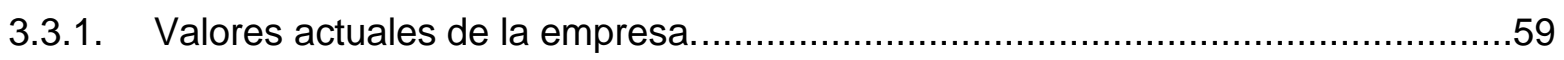

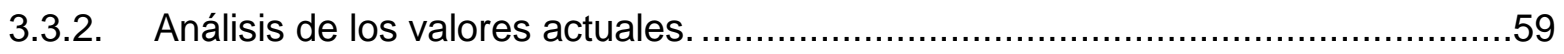

3.3.3. Elementos de los valores propuestos para la empresa................................59

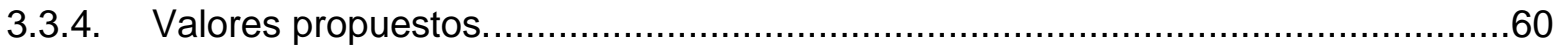

3.3.5. Elementos de los valores propuestos agua mineral San Mateo.........................60

3.4. Alineamiento estratégico de la Misión, Visión y Valores de la empresa Backus.............60

3.5. Alineamiento estratégico de la Misión, Visión y Valores de agua mineral San Mateo ....61

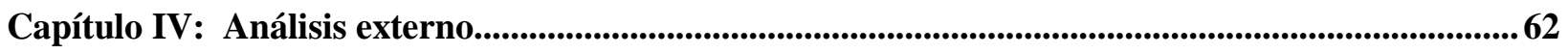

4.1. Prospectiva del Entorno: Megatendencias ............................................................62

4.2. Tendencias de las variables del entorno ...................................................65

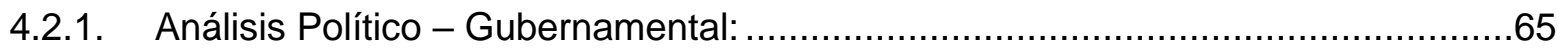

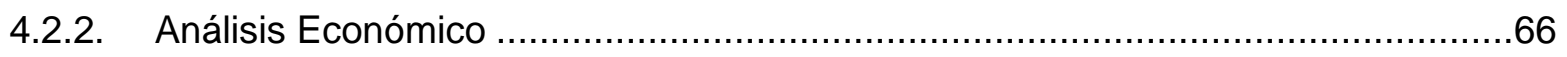

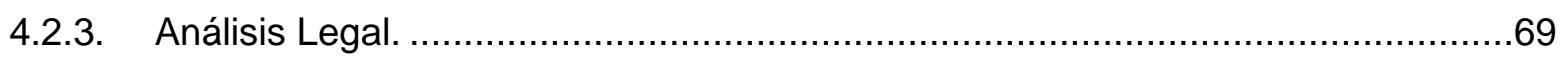

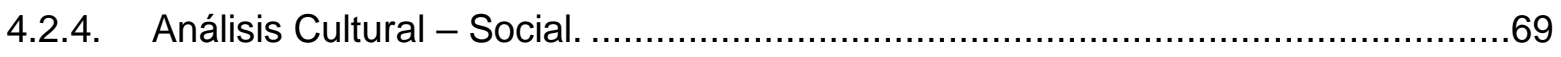

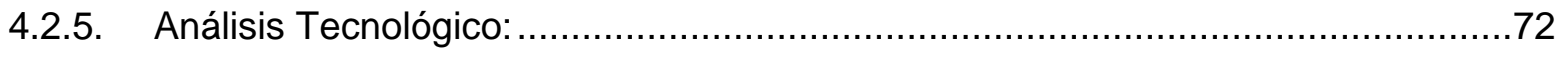

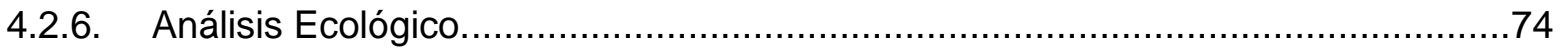

4.3. Efecto de las variables del entorno sobre la empresa: .....................................75 
4.4. Impacto en clientes / proveedores de cada una de las variables del entorno .78

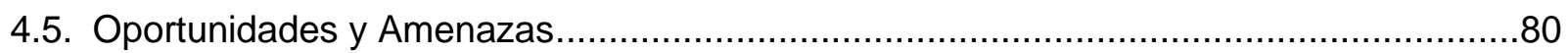

4.6. Matriz de Evaluación de los Factores Externos EFE $\ldots \ldots \ldots \ldots \ldots \ldots \ldots \ldots \ldots \ldots \ldots \ldots \ldots \ldots . . . . \ldots 1$

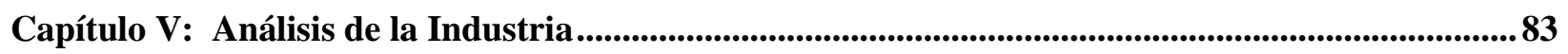

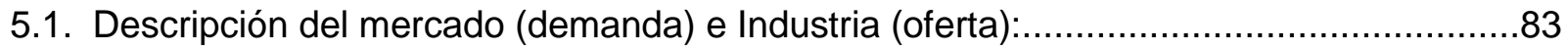

5.2. Descripción de las cinco fuerzas competitivas de la industria: ...............................91

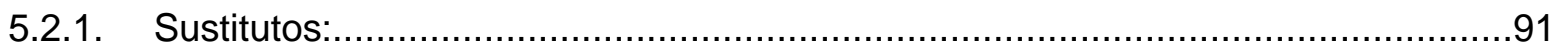

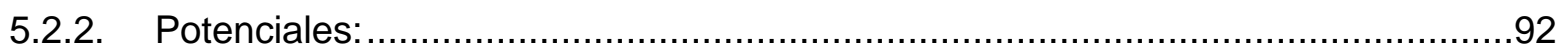

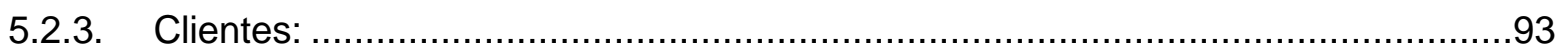

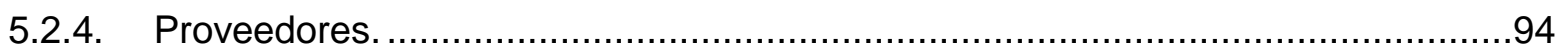

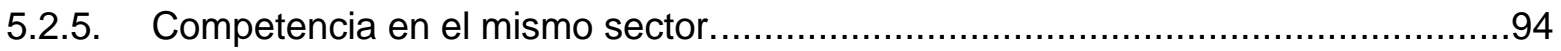

5.3. Matriz de atractividad de cada una de las cinco fuerzas ..........................................95

5.4. Análisis del grado de atractividad de la industria ..........................................98

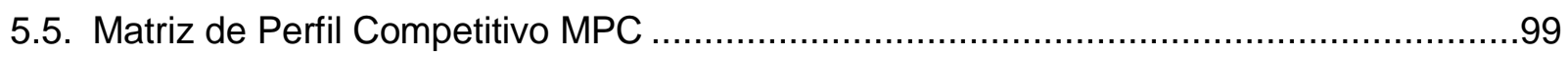

Capítulo VI: Análisis Interno.....................................................................................................................................101

6.1. Descripción de las actividades de la cadena de valor de la empresa:......................101

6.2. Indicadores de cada una de las actividades de la cadena de valor ..........................106

6.3. Benchmarking entre Backus y Lindley. Comparación con los líderes de la industria en cada una de las actividades de la cadena de valor ..............................................

6.4. Determinar las competencias de la empresa Backus.......................................110

6.5. Identificación y determinación de las ventajas competitivas de la empresa ................113

6.6. Análisis interno de agua mineral San Mateo ..................................................114

6.7. Matriz de Evaluación de los Factores Internos EFI ..........................................124 
Capítulo VII: Formulación de los objetivos y diseño de las estrategias..........................................126

7.1. Alcance y Planteamiento de los Objetivos Estratégicos .......................................126

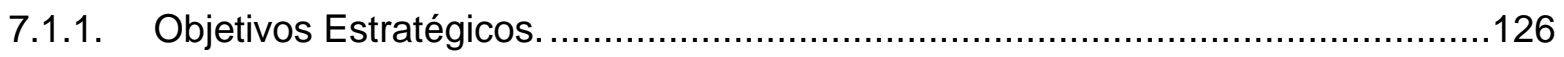

7.1.2. Análisis de los Objetivos Estratégicos .................................................128

7.2. Diseño y Formulación de Estrategias ............................................................130

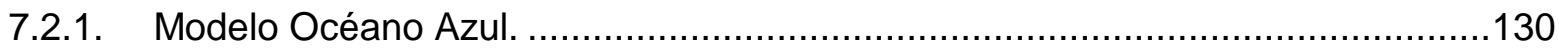

7.2.1.1. Lienzo de la estrategia actual de la empresa...................................131

7.2.1.2. Lienzo de la estrategia de la industria.............................................131

7.2.1.3. Matriz (eliminar, reducir, incrementar, crear) ................................... 132

7.2.1.4. Lienzo de la nueva estrategia considerada.....................................133

7.2.2. Matrices de formulación de estrategias ....................................................134

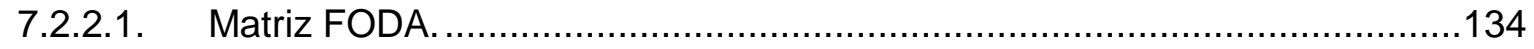

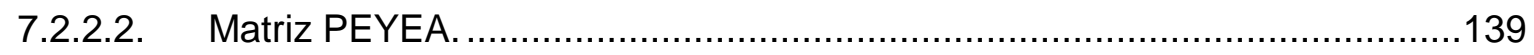

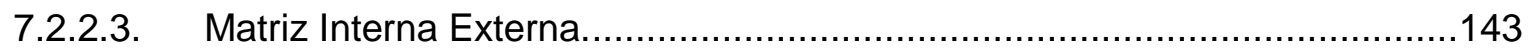

7.2.2.4. Matriz Boston Consulting Group. ............................................... 146

7.2.2.5. Matriz de la Gran Estrategia. ...................................................148

7.3. Resumen de las Estrategias Formuladas .........................................................150

Capítulo VIII: Selección de la Estrategia ..........................................................................................153

8.1. Métodos Factores Estratégicos Claves ......................................................153

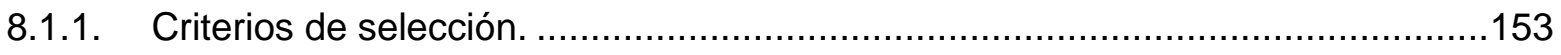

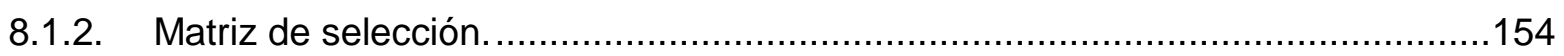

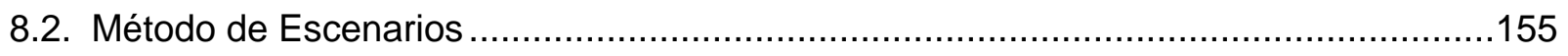

8.2.1. Descripción de escenarios considerados...................................................156

8.2.1.1. E2 Estrategia de Diferenciación...........................................................156

8.2.1.2. E3 Estrategia de Penetración. ........................................................156

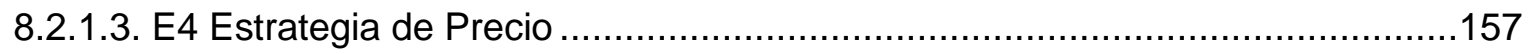


8.2.2. Comparación de estrategias con escenarios. .........................................157

8.3. Matriz de Planeación Estratégica Cuantitativa MPEC ............................................159

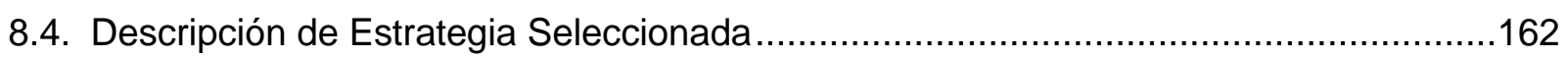

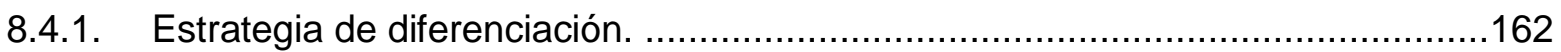

8.4.2. Estrategia de penetración de mercado.....................................................162

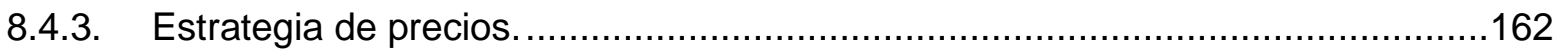

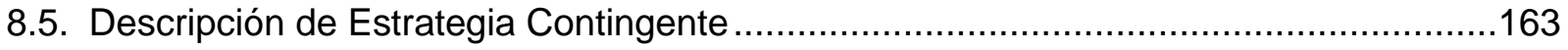

Capítulo IX: Implantación de la estrategia .........................................................................................165

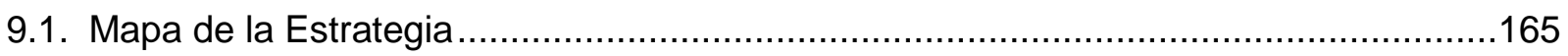

9.2. Objetivos específicos según el mapa de la estrategia..........................................166

9.3. Indicadores para cada uno de los objetivos específicos .......................................168

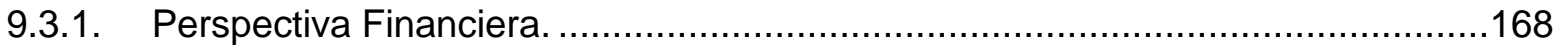

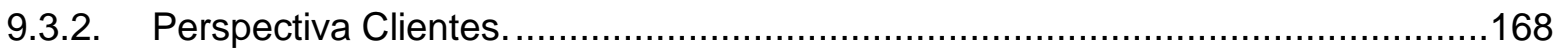

9.3.3. Perspectiva Procesos Internos. ........................................................168

9.3.4. Perspectiva Aprendizaje y Crecimiento. ...............................................169

9.4. Metas para cada uno de los objetivos específicos ..........................................169

9.4.1. Perspectiva Financiera. .......................................................................... 169

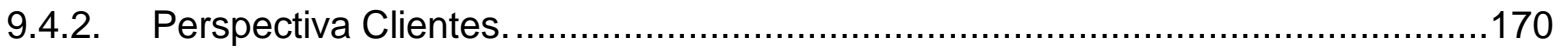

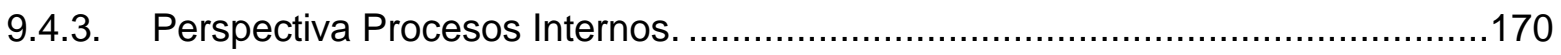

9.4.4. Perspectiva Aprendizaje y Crecimiento. .............................................170

9.5. Iniciativas, Estrategias, Programas, Políticas, Reglas, Procedimiento .......................171

9.6. Responsable de cada una de las iniciativas ..............................................173

9.7. Presupuesto de cada una de las iniciativas ...................................................173

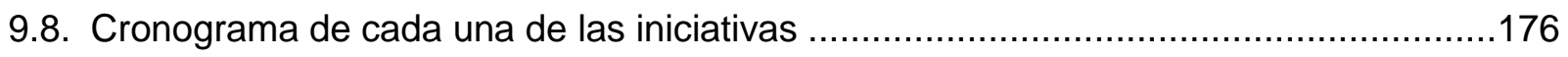




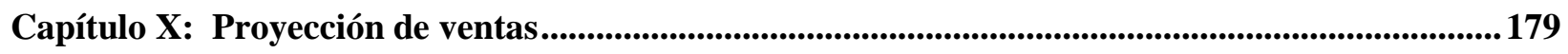

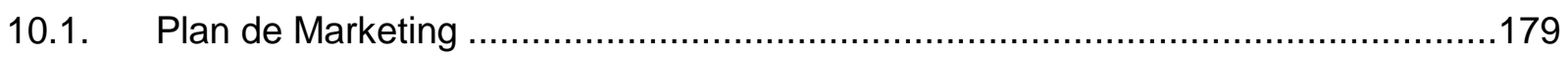

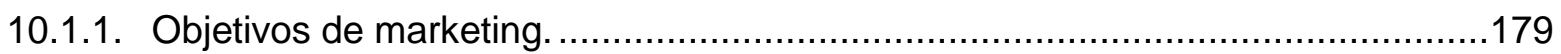

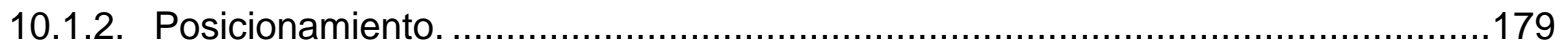

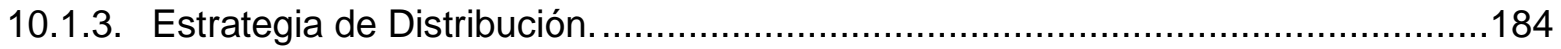

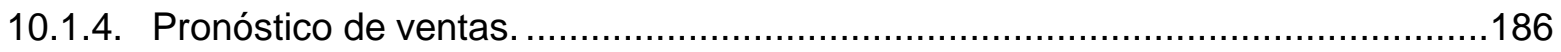

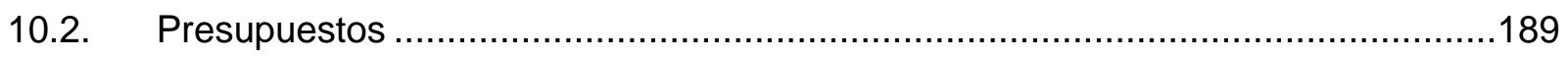

Capítulo XI: Evaluación.................................................................................................................................................... 194

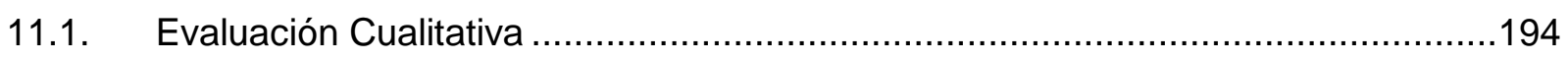

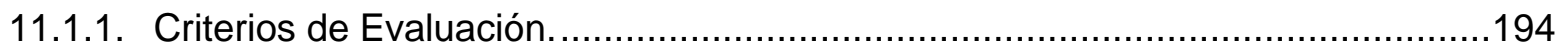

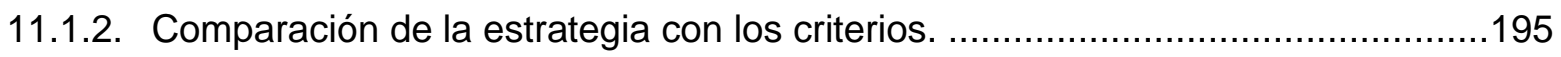

11.2. Evaluación Financiera de la Estrategia............................................................196

11.2.1. Proyección de estados financieros. ..................................................... 196

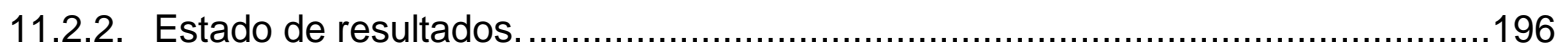

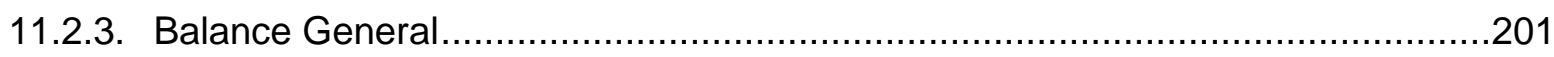

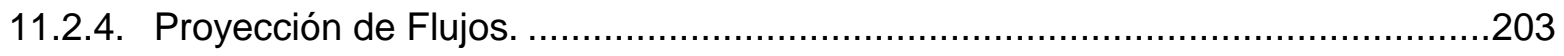

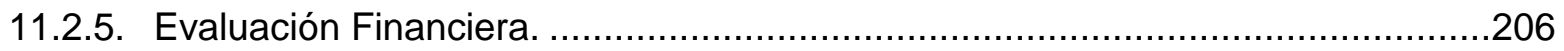

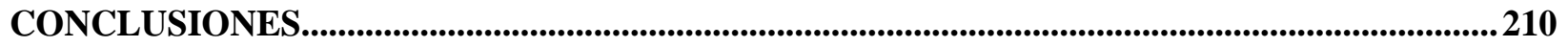

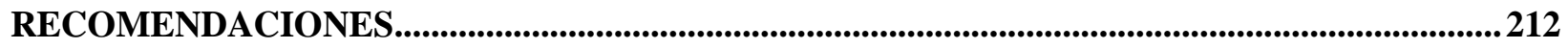

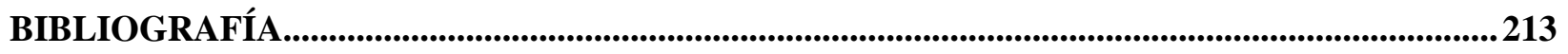

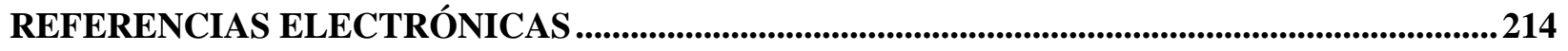

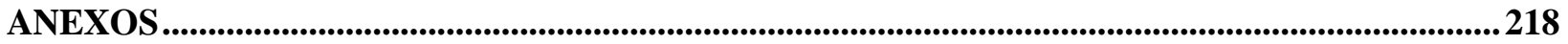




\section{Lista de Tablas}

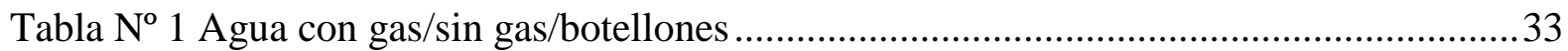

Tabla $\mathrm{N}^{\circ} 2$ Características del ciclo de vida del producto ……….............................................. 37

Tabla N 3 Comparativo Cifras Financieras Backus - Lindley año 2014 vs. año 2013............46

Tabla No 4 Porcentaje de Participación de Mercado de Agua San Mateo en la industria. .......46

Tabla N 5 Ventas Totales por Categoría de Backus año 2014 ...............................................4

Tabla No 6 Ventas Valorizadas por categoría de Backus año 2014 ……………………….......4

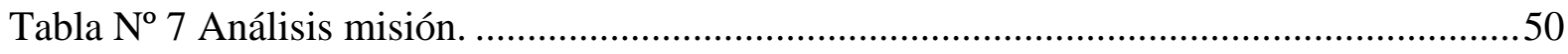

Tabla No 8 Características de la misión - empresa Backus.......................................................51

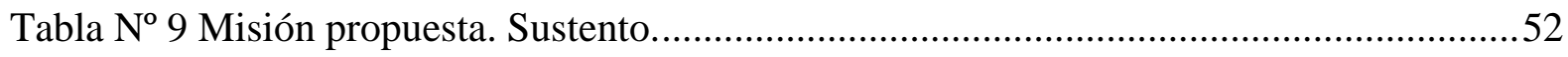

Tabla N 10 Misión propuesta. Razones..............................................................................53

Tabla $N^{\circ} 11$ Preguntas para formular la misión de agua mineral San Mateo.............................54

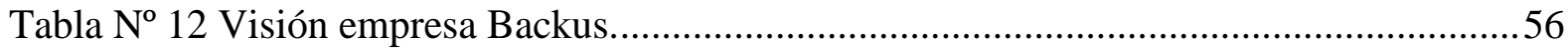

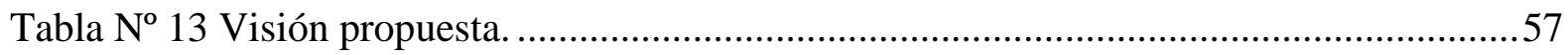

Tabla N 14 Visión propuesta para agua mineral San Mateo. ....................................................58

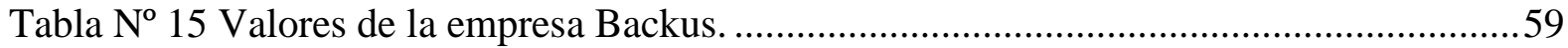

Tabla No 16 Valores propuestos para agua mineral San Mateo................................................ 60

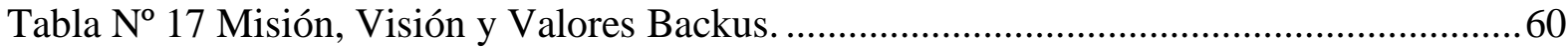

Tabla N ${ }^{\circ} 18$ Misión, Visión y Valores para agua mineral San Mateo.......................................61

Tabla $\mathrm{N}^{\mathrm{o}} 19$ Impacto de las Megatendencias en la empresa...................................................64

Tabla $\mathrm{N}^{\circ} 20$ Impacto de las Variables Externas en la Empresa..............................................75

Tabla N 21 Impacto de las Variables Externas en los Clientes y Proveedores. ........................78

Tabla $\mathrm{N}^{\mathrm{0}} 22$ Identificación de las Oportunidades y Amenazas..................................................8 80 


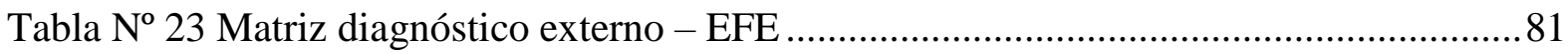

Tabla $\mathrm{N}^{\circ} 24$ Nivel de atractividad de competidores indirectos..............................................95

Tabla $\mathrm{N}^{\circ} 25$ Nivel de atractividad de competidores potenciales. ............................................96

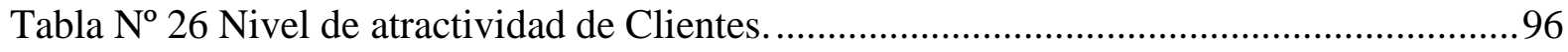

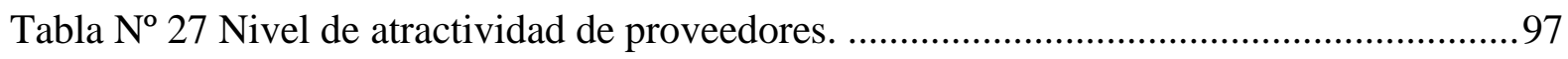

Tabla $\mathrm{N}^{\circ} 28$ Nivel de atractividad de competencia en el mismo sector...................................98

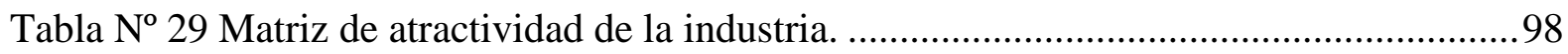

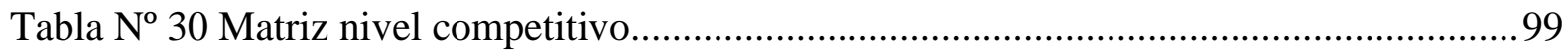

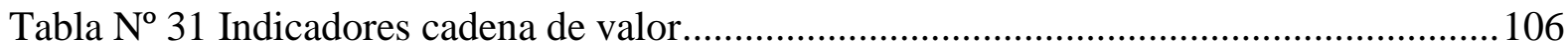

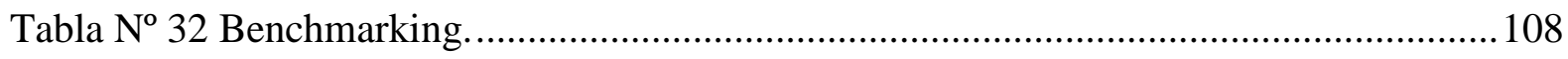

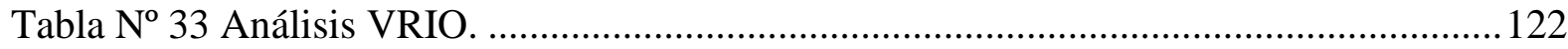

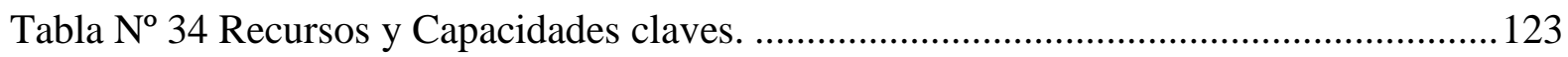

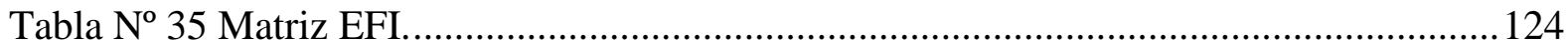

Tabla No 36 Esquema actual de competencia en el sector.................................................... 131

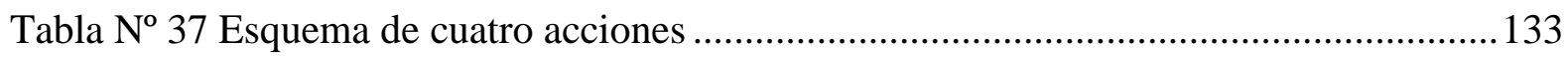

Tabla $\mathrm{N}^{\circ} 38$ Esquema propuesto de competencia en el sector..............................................134

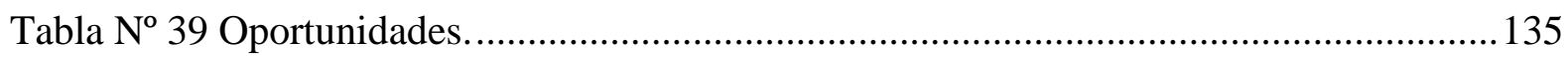

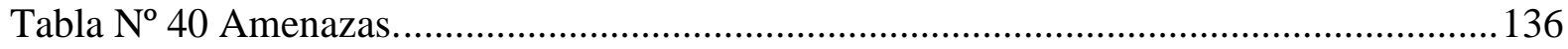

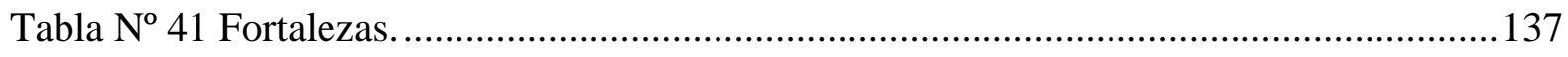

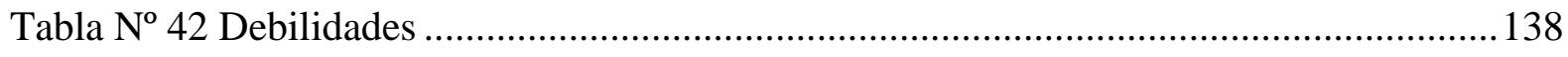

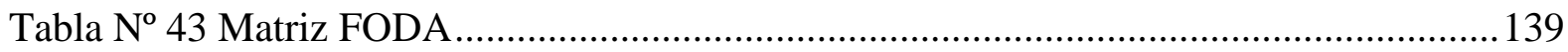

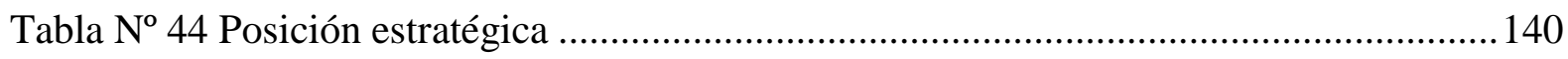

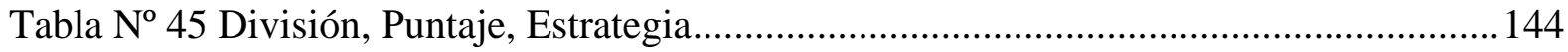


Tabla $N^{\circ} 46$ Indicadores de ventas y financieros de la empresa Backus 147

Tabla Nº 47 Matriz de Selección. 154

Tabla No 48 Estrategia. Escenario positivo. Escenario negativo. 157

Tabla No 49 Matriz diagnóstico externo. 159

Tabla N ${ }^{\circ} 50$ Consecuencias versus Probabilidad. 163

Tabla $N^{\circ} 51$ Estrategia de contingencia 164

Tabla No 52 Objetivos estratégicos. Objetivos específicos. Perspectiva. 167

Tabla N 53 Objetivos estratégicos por Perspectiva. 171

Tabla $N^{\circ} 54$ Iniciativas estratégicas. 173

Tabla No 55 Iniciativas por implementar. 174

Tabla $N^{\circ} 56$ Cronograma de iniciativas. 176

Tabla N 57 Canales de distribución San Mateo. 184

Tabla No 58 Pronóstico de Ventas 186

Tabla N 59 Producción aguas 2013 - 2015 (en Hectolitros). 187

Tabla N ${ }^{\circ} 60$ Venta anual proyectada Año 1 (en Hectolitros). 187

Tabla N ${ }^{\circ} 61$ Venta anual proyectada Año 2 (en Hectolitros) 188

Tabla N ${ }^{\circ} 62$ Venta anual proyectada Año 3 (en Hectolitros) 189

Tabla No 63 Matriz de Rumelt. 195

Tabla $N^{\circ} 64$ Estado de resultados sin estrategia 198

Tabla $N^{\circ} 65$ Estado de resultados con estrategia 199

Tabla Nº 66 Balance General 2015. 202

Tabla $\mathrm{N}^{\mathrm{o}} 67$ Aporte a la cuenta de resultados acumulados. 203

Tabla $N^{\circ} 68$ Flujo de caja proyectado sin estrategia 204 
Tabla N ${ }^{o} 69$ Flujo de caja proyectado con estrategia...................................................205

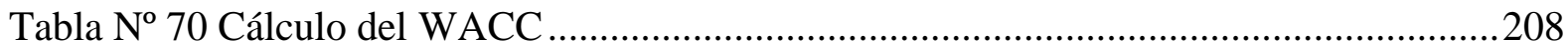

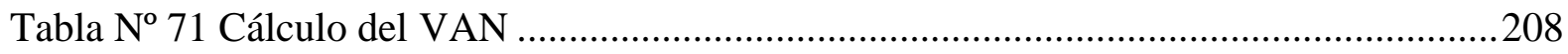

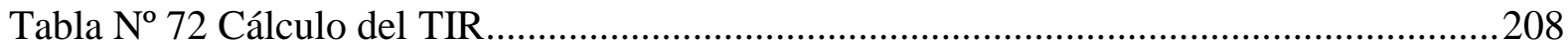

\section{Lista de Figuras}

Figura $\mathrm{N}^{\mathrm{o}}$ 1. Evolución del mercado total de bebidas...................................................20

Figura $\mathrm{N}^{\mathrm{o}}$ 2. Comparativa atributos diferenciadores - Aguas .......................................... 24

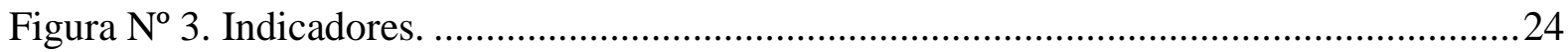

Figura $N^{o}$ 4. Imágenes de agua mineral San Mateo.....................................................2 27

Figura $\mathrm{N}^{\mathrm{o}}$ 3. Participación nacional de aguas en volumen............................................... 32

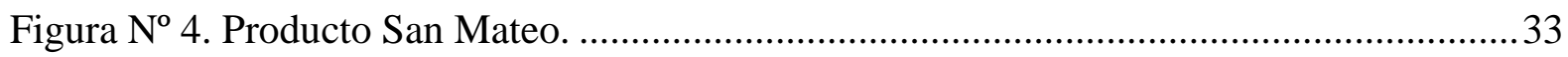

Figura $\mathrm{N}^{\circ}$ 5. Imágenes de agua mineral San Mateo............................................................ 34

Figura $N^{\circ}$ 6.: Producción entre los años 2012 al 2015.................................................. 36

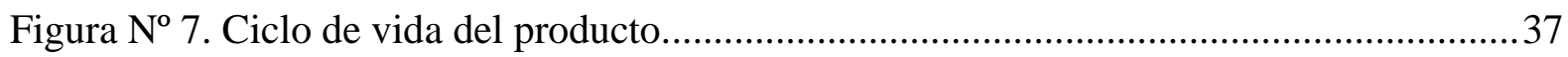

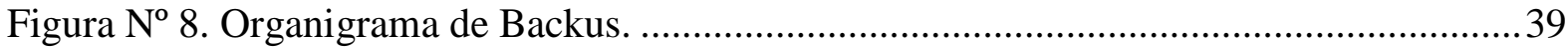

Figura $\mathrm{N}^{\mathrm{o}}$ 9. Penetración bebida no alcohólicas. ........................................................ 44

Figura $\mathrm{N}^{\mathrm{o}}$ 10. Crecimiento anual del consumo de aguas versus gaseosas...........................45

Figura $\mathrm{N}^{\mathrm{o}}$ 11. Participación nacional de aguas en volumen............................................ 47

Figura $\mathrm{N}^{\mathrm{o}}$ 12. Canales de ventas de aguas en valor. .................................................... 48

Figura $N^{\circ}$ 13. Producto Bruto Interno y Demanda Interna. .............................................67

Figura $\mathrm{N}^{\mathrm{0}}$ 14. Visualización del Perú - Ejecutivos 2015...............................................6 68

Figura N 15. Proyección sobre visualización del Perú a ejecutivos, 2016 versus 2015........68 


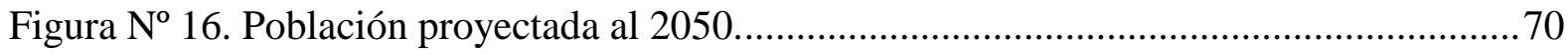

Figura $\mathrm{N}^{\circ}$ 17. Población con conocimiento financiero. ........................................................

Figura $\mathrm{N}^{\mathrm{o}}$ 18. Población de las principales ciudades del país....................................................71

Figura $\mathrm{N}^{\mathrm{0}}$ 19. Distribución hogares por NSE.....................................................................

Figura $\mathrm{N}^{\circ}$ 20. Investigadores por cada mil integrantes de la PEA.........................................73

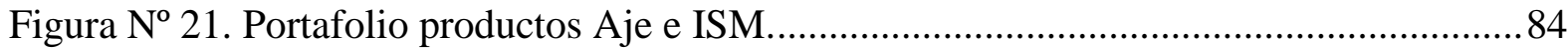

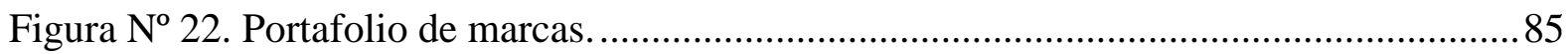

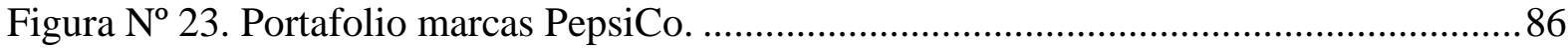

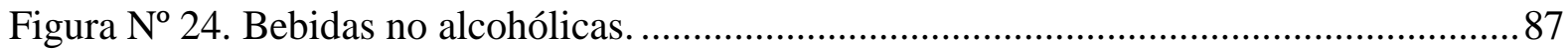

Figura $\mathrm{N}^{\mathrm{0}}$ 25. Penetración de bebidas no alcohólicas nacional.................................................. 88

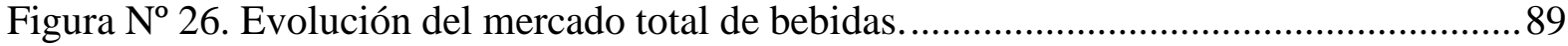

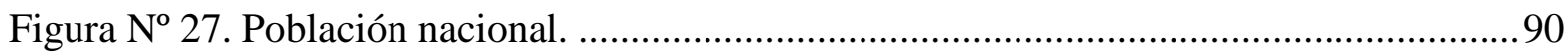

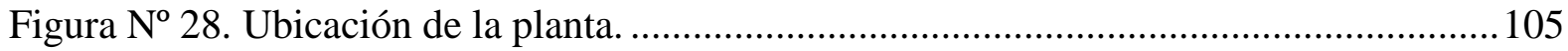

Figura $\mathrm{N}^{\mathrm{o}}$ 29. Participación nacional. Share of volumen....................................................... 114

Figura $\mathrm{N}^{\mathrm{o}}$ 30. Participación nacional. Share of value. …….................................................... 115

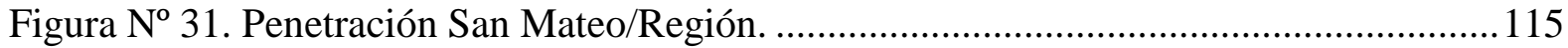

Figura $\mathrm{N}^{\circ}$ 32. Evolución de ventas por región (kHl) (Participación)........................................116

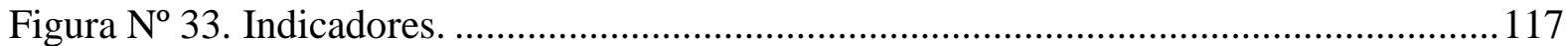

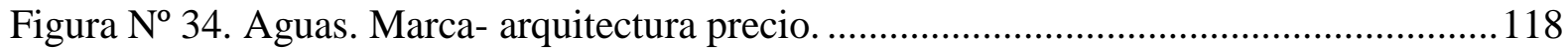

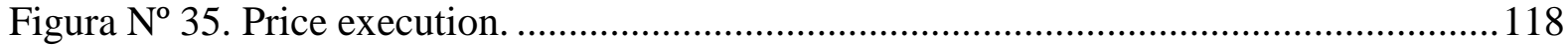

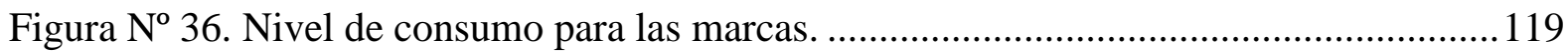

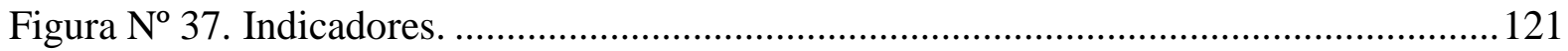

Figura $\mathrm{N}^{\mathrm{o}}$ 38. Lienzo de agua San Mateo en el sector. .........................................................132 
Figura $\mathrm{N}^{\text {o } 39 .}$ Lienzo de la nueva estrategia de Agua Mineral San Mateo........................... 134

Figura $\mathrm{N}^{\mathrm{0}}$ 40. Representación gráfica del resultado de la Matriz PEYEA. .......................... 142

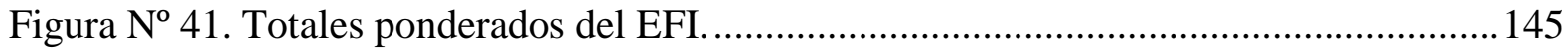

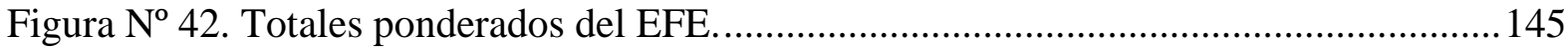

Figura $N^{o}$ 43. Posición de la participación relativa en el mercado. .................................... 147

Figura $N^{\circ}$ 44. Matriz de la gran estrategia para la empresa Backus. .................................. 149

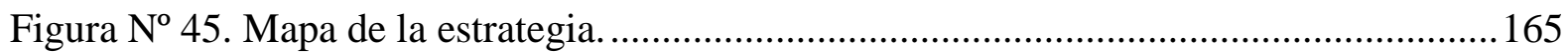

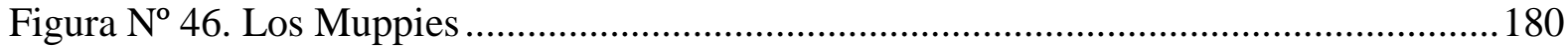

Figura $N^{\circ}$ 47. Atributos de agua mineral San Mateo. ........................................................ 181

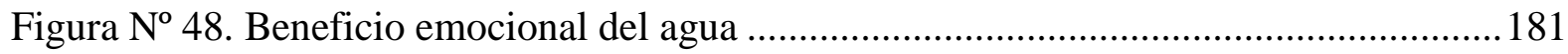

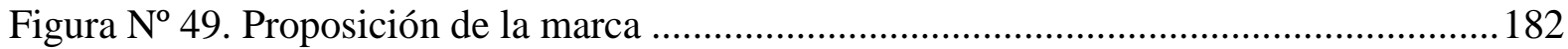

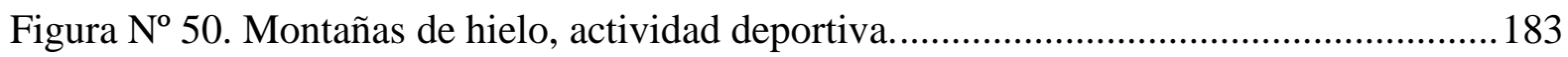

Figura $\mathrm{N}^{\mathrm{o}}$ 51. Rango de precio en porcentaje ........................................................ 183

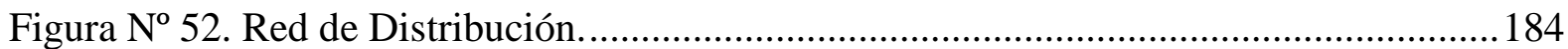




\section{INTRODUCCIÓN}

A inicios del año 2013 se identificó una creciente migración del consumo hacia los productos más saludables, como jugos, bebidas isotónicas, té, bebidas vitaminadas y aguas embotelladas. Durante el año 2014, el sector de bebidas no alcohólicas mostró un ligero crecimiento en términos de volumen, particularmente de las bebidas diferentes a gaseosas. El mercado total de bebidas está compuesto por refrescos, jugos, néctares, energizantes, aguas y gaseosas. En el año 2015 el mercado total de bebidas creció en 3\%, sin embargo, la categoría de aguas obtuvo un crecimiento del 14\%, restándole participación a los refrescos y bebidas gaseosas.

La elaboración del presente trabajo ha seguido la metodología expuesta en clase por los docentes del curso de Planeamiento Estratégico. Del mismo modo, los temas desarrollados siguen la estructura propuesta por la Escuela de Postgrado.

El capítulo uno del trabajo explora la oportunidad de negocio que ofrece el mercado nacional de bebidas, de manera particular el agua embotellada - el mismo que registra un crecimiento del sector favorecido por el interés en el cuidado de la salud -, para luego dar lugar a la descripción del problema y al sustento de la justificación del presente trabajo.

El capítulo dos está dedicado a proporcionar una descripción general de la empresa y del negocio, en tanto que el capítulo tres hace el análisis de la declaración de visión, misión y valores de la empresa con el objetivo de alinearlos con la propuesta de valor desarrollada en este trabajo.

El capítulo cuatro realiza un análisis del entorno político, legal, económico, cultural, etc., en el que opera la empresa. Esto se hace con el objetivo facilitar la posterior identificación de las fuerzas externas que influyen en el mercado. A continuación, en el capítulo cinco se desarrolla un análisis de la industria nacional de bebidas tomando como base la información registrada en el capítulo anterior y aplicando el modelo de las cinco fuerzas competitivas de Michael Porter. 
En el capítulo seis se describen las actividades que integran la cadena de valor de la empresa y que constituyen sus principales competencias de mercado. En tanto que el capítulo siete se encarga del diseño de las matrices que contribuirán a la formulación de las estrategias desarrolladas en este trabajo (FODA, PEYEA, IE, BCG, Matriz de la estrategia principal).

El capítulo ocho se encarga de la selección de las estrategias que deben ser consideradas por la empresa, utilizando para ello la matriz de planeación estratégica cuantitativa. Por su parte, el capítulo nueve aborda el desarrollo del mapa estratégico, el cual determinará los lineamientos que deberá seguir la empresa.

El capítulo diez describe el plan de marketing, la proyección de ventas y el presupuesto, información sobre la cual se basa el capítulo once para desarrollar el análisis financiero del presente trabajo. En este análisis se incluyen los principales estados financieros (Balance General, Estado de Resultados, Flujos de caja y Ratios financieros).

En el capítulo doce se presentan las conclusiones y recomendaciones finales que son el resultado del desarrollo del presente trabajo.

En la parte final del trabajo se encuentran los anexos citados a lo largo del desarrollo del trabajo. 